Noname manuscript No.

(will be inserted by the editor)

\title{
Landau quasiparticles in weak power-law liquids
}

\author{
Joshuah T. Heath
}

Received: date / Accepted: date

\begin{abstract}
The failure of Landau-Fermi liquid theory is often considered a telltale sign of universal, scale-invariant behavior in the emergent field theory of interacting fermions. Nevertheless, there exist borderline cases where weak scale invariance coupled with particle-hole asymmetry can coexist with the Landau quasiparticle paradigm. In this letter, I show explicitly that a Landau-Fermi liquid can exist for weak power-law scaling of the retarded Green's function. Such an exotic variant of the traditional Fermi liquid is shown to always be incompatible with Luttinger's theorem for any non-trivial scaling. This result yields evidence for a Fermi liquid-like ground state in the high-field, underdoped pseudogap phase of the high-temperature cuprate superconductors.
\end{abstract}

Keywords Landau-Fermi liquids · Non-Fermi liquids · Quasiparticle methods · Luttinger's theorem

J.T. Heath

Physics Department

Boston College

140 Commonwealth Avenue

Chestnut Hill, MA 02467

Tel.:+802-922-1291

E-mail: heathjo@bc.edu 
I. Introduction.--In recent years, the study of non-Fermi liquids has been dominated by quantum critical phenomena [1,2, 3, 4, 5. In particular, the transport quantities of the optimally-doped cuprate superconductors are now known to exhibit quantum critical scaling, with the the electrical resistivity [6,7,8, the Hall angle [9, 10], and the Lorentz ratio [11] all exhibiting temperature dependencies inconsistent with super-Planckian lifetimes of the charge carriers [12, 13. Nevertheless, the underlying microscopic origin of such behavior is still a matter of debate, with proposals ranging from coupled SYK islands [14, 15, 16, to an exotic phase of matter which lacks charge quantization [17, 18,

Mirroring the microscopic underpinnings of conventional Landau-Fermi liquids [19, 20,21,22], Anderson first proposed that the unusual normal phase of the optimally-doped high- $T_{c}$ cuprates can be explained via a "hidden Fermi liquid theory" 23,24]. The Fourier-transformed Green's function of such a system can be written as a Landau-like propagator raised to a power proportional to the sum of phase shifts over all scattering channels, reducing the quasiparticle pole to a branch cut. Such "power-law liquids" 17 have recently been shown to violate the Luttinger sum rule [26], and interact with conventional electrons in such a way that may lead to a power-law scaling of the imaginary self energy similar to what is seen in ARPES experiments on $\mathrm{Bi}_{2} \mathrm{Sr}_{2} \mathrm{CaCu}_{2} \mathrm{O}_{8+\delta}$ in the underdoped pseudogap phase 25, 27, 28. Indeed, such power-law behavior is often taken as the hallmark of an "unparticle"-like phase, where scale invariance naturally leads to a power-law Green's function and the Standard Model notion of an independent particle breaks down [29, 30. Nevertheless, there have been several cases in the past few years that have shown that Landau-Fermi liquid theory persists in close proximity to a scaleinvariant quantum critical point $[31,32,33,34$, contradicting the underlying predictions of an unparticle-like condensate.

In this letter, I will show that power-law scaling of the electronic Green's function does not necessarily imply a non-trivial IR fixed point in the manybody field theory. Specifically, in dimensions $d \geq 2$, the spectral density exhibits a clearly defined peak and weight for Green's functions raised to reasonably small positive powers. The existence of a finite quasiparticle weight is shown to always be incompatible with Luttinger's theorem for all non-zero powers, supporting the claim that the underdoped pseudogap phase of the cuprates at high magnetic fields [35] may be a Luttinger's theorem-violating Landau-Fermi liquid [36] or "fractionalized" Fermi liquid [37,38, 39, 40].

II. Spectral density of power-law Green's functions.- I consider Green's functions for interacting fermions raised to some power $\xi \in \mathbb{R}$ :

$$
G_{\xi}(\mathbf{k}, \omega)=\frac{1}{\left(\omega-\epsilon_{\mathbf{k}}-\Sigma(\mathbf{k}, \omega)+i \delta\right)^{1+\xi}}
$$

\footnotetext{
1 Although power-law liquids were originally defined as a system characterized by a powerlaw scaling of the self energy 25, I take the terminology to mean some general scaling of the Green's function itself [26] throughout this paper.
} 
where the self energy $\Sigma(\mathbf{k}, \omega)$ is given by $\Sigma(\mathbf{k}, \omega)=\left(G_{0}^{(0)}\right)^{-1}-G_{0}^{-1}$. If $\xi=0$, we obtain the well-known propagator of the dressed Landau quasiparticle, with the presence of a finite pole dictated by the frequency-dependence of the real part of the self energy [20,41,42].

If $\xi \neq 0$, it is often claimed that the resultant branch cut kills off any welldefined quasiparticle, but this claim is misguided. The oft-quoted example of such a power-law non-Fermi liquid is the 1D Tomonaga-Luttinger model 43 , 44,45,46, which is described by a propagator of the form

$$
G_{ \pm}(\mathbf{k}, \omega)=\frac{\left(\widetilde{v}_{F}^{2} k^{2}-\omega^{2}\right)^{s^{2} / 2-1}}{\left|\left( \pm \widetilde{v}_{F} k-\omega\right)\left( \pm v_{F} k-\omega\right)\right|^{1 / 2}}
$$

where $\widetilde{v}_{F}$ and $s$ are dependent on the interaction and \pm denote right- and left-handed fermions, respectively. However, it is crucial to note that branch cut singularities for the Tomonaga-Luttinger system exist on either side of the Fermi point by virtue of the $g$-ology construction of the 1D Hamiltonian 41. For higher-dimensional systems characterized by Green's functions such as Eqn. (1), we can simply close the contour on the opposite side of the realfrequency axis to preserve the pole structure [4], assuming that the branch cut does not include the singularity of the Green's function (i.e., the branch cut does not include the Fermi point/surface). Physically, when the branch cut replaces the pole, the bare electron distribution function is characterized by an infinite slope as opposed to a finite discontinuity, resulting in either Luttinger liquid or "marginal Fermi liquid" behavior [47,48. In the case where branch cuts coexist with poles, however, the Fermi liquid picture has been shown to remain, albeit with non-analyticities in the thermodynamic potential [49. In a similar fashion, a higher-dimensional system composed of Tomonaga-Luttinger "threads" coupled via a Coulomb interaction display power-law behavior in the electron propagator, yet exhibits a finite discontinuity in the momentum dispersion unseen in the "true" 1D model [50. It should therefore be apparent that the breakdown of Landau-Fermi liquid theory in one dimension (and fractional dimensions $1<d<2$ [51] ) is an inherent consequence of dimensional reduction, and not from the appearance of a branch cut from the power-law nature of the propagator.

To investigate the possibility of Landau-like excitations in the power-law liquid, I solve for the spectral density $A(\mathbf{k}, \omega) \sim \Im G_{\xi}(\mathbf{k}, \omega)$ resulting from Eqn. (1). To do this, I simplify the power-law Green's function via the following:

$$
\begin{aligned}
G(k, \omega) & =\frac{1}{\left(\omega-\epsilon_{k}-\Sigma(\mathbf{k}, \omega)+i \delta\right)^{1+\xi}} \\
& =\sum_{n=0}^{\infty} \frac{(-\xi)^{n}}{n !} \frac{\left(\log \left(\omega-\epsilon_{k}-\Sigma(\mathbf{k}, \omega)+i \delta\right)\right)^{n}}{\omega-\epsilon_{k}-\Sigma(\mathbf{k}, \omega)+i \delta}
\end{aligned}
$$

This allows us to write the spectral function in the form 


$$
\begin{aligned}
A \sim & -\frac{1}{\pi} \frac{\Im \Sigma(\mathbf{k}, \omega)}{(x-\Re \Sigma(\mathbf{k}, \omega))^{2}+\Im \Sigma(\mathbf{k}, \omega)^{2}} \\
& \times \sum_{n=0}^{\infty} \frac{(-\xi)^{n}}{n !}\left\{r^{n}\left(\cos (n \theta)+\frac{x-\Re \Sigma(\mathbf{k}, \omega)}{\Im \Sigma(\mathbf{k}, \omega)} \sin (n \theta)\right)\right\}
\end{aligned}
$$

where $x \equiv \omega-\epsilon_{\mathbf{k}}$ and $r$ and $\theta$ are the modulus and phase of the complex logarithm in Eqn. (3), respectively. We can simplify further by performing each sum separately and recalling the phase $\phi_{0}(\omega)$ of the retarded Green's function for $\xi=0$ :

$$
\tan \phi_{0}(\omega)=\frac{\Im\left(G_{0}(k, \omega)\right)}{\Re\left(G_{0}(k, \omega)\right)}=\frac{\Im \Sigma(\mathbf{k}, \omega)}{x-\Re \Sigma(\mathbf{k}, \omega)}
$$

The behavior of such a phase is central to the discussion of Luttinger's theorem in these power-law liquids, which is the topic of the next section.

The above allows us to write the spectral function in the form

$$
\frac{A_{\xi}(k, \omega)}{A_{0}(k, \omega)}=\frac{\cos \left[\xi \phi_{0}(\omega)\right]-\cot \left[\phi_{0}(\omega)\right] \sin \left[\xi \phi_{0}(\omega)\right]}{\left((x-\Re \Sigma(\mathbf{k}, \omega))^{2}+\Im \Sigma(\mathbf{k}, \omega)^{2}\right)^{\xi / 2}}
$$

where I take the notation $A_{\xi}(\mathbf{k}, \omega)$ to mean the spectral function at some non-zero power $\xi$ and $A_{0}(\mathbf{k}, \omega)$ to be the spectral function for trivial power $\xi=0$. Note that, as $\xi \rightarrow 0$, the right-hand side approaches unity, as expected.

With the spectral function written as Eqn. (6), we can now write down an approximate form near the Fermi surface:

$$
\begin{aligned}
A_{\xi}(k, \omega) & =A_{0}(k, \omega)\left\{\frac{\cos \left[\xi \phi_{0}(\omega)\right]-\cot \left[\phi_{0}(\omega)\right] \sin \left[\xi \phi_{0}(\omega)\right]}{\left(\left(x-\Sigma^{\prime}\right)^{2}+\Sigma^{\prime \prime 2}\right)^{\xi / 2}}\right\} \\
& \sim-\frac{1}{\pi} \frac{\Sigma^{\prime \prime} Z_{k}^{2}}{\widetilde{x}^{2}+\Sigma^{\prime \prime 2} Z_{k}^{2}}\left\{Z_{k}^{\xi} \frac{\cos \left[\xi \phi_{0}(\omega)\right]-\cot \left[\phi_{0}(\omega)\right] \sin \left[\xi \phi_{0}(\omega)\right]}{\left(\widetilde{x}^{2}+\Sigma^{\prime \prime 2} Z_{k}^{2}\right)^{\xi / 2}}\right\}+A_{i n c} \\
& \sim \frac{\widetilde{Z}_{k}}{\pi} \frac{1 / \tau}{\left(\widetilde{x}^{2}+(1 / \tau)^{2}\right)^{1+\xi / 2}}+A_{i n c}
\end{aligned}
$$

where $A_{\text {inc }}$ is the incoherent part of the spectral weight, $\tau$ is the width of the spectral density for $\xi=0$, and the "effective" quasiparticle weight is given by

$$
\widetilde{Z}_{k}=Z_{k}^{1+\xi}\left\{\cos \left[\xi \phi_{0}(0)\right]-\cot \left[\phi_{0}(0)\right] \sin \left[\xi \phi_{0}(0)\right]\right\}
$$

where $Z_{k}$ is the conventional quasiparticle weight for a Fermi liquid at $\xi=0$, given by

$$
Z_{k}=\left(1-\left.\frac{\partial \Re \Sigma(\mathbf{k}, \omega)}{\partial \omega}\right|_{\omega=0}\right)^{-1}
$$


The above formulation of the power-law Green's function therefore allows us to recast this system in terms of a conventional, Fermi liquid-like Green's function with the power-law dependence buried in a re-scaled quasiparticle residue:

$$
\begin{aligned}
G^{R} & =\int_{-\infty}^{\infty} d \omega^{\prime} \frac{A_{\xi}(\mathbf{k}, \omega)}{\omega-\omega^{\prime}+i \delta} \\
& =\int_{-\infty}^{\infty} d \omega^{\prime} \frac{1}{\omega-\omega^{\prime}+i \delta}\left\{\frac{\widetilde{Z}_{k}}{\pi} \frac{1 / \tau}{\left(\widetilde{x}^{2}+(1 / \tau)^{2}\right)^{1+\xi / 2}}+A_{i n c}\right\} \\
& =\frac{\widetilde{Z}_{k}}{\widetilde{x}-\frac{i}{\tau_{k}}}+G_{i n c}^{R}
\end{aligned}
$$

The dependence on $\xi$ in the "effective" quasiparticle weight tells us that nontrivial power-law scaling of the Green's function scales the discontinuity of the electronic distribution function in a non-trivial way. Later on, we will see that a finite subset of powers $\xi$ will result in a stable Fermi liquid; i.e., a value $0<\widetilde{Z}_{k} \leq 1$.

The final form of the spectral weight can be found by imposing the sum rule $\int_{-\infty}^{\infty} A(\mathbf{k}, \omega)=1$ on Eqn. (7). This follows from the fact that $A(\mathbf{k}, \omega)$ can still be interpreted as probability density for non-trivial powers $\xi>-1$ (as previously shown in [26]), and yields the appropriate normalization constant:

$$
A_{\xi}(\mathbf{k}, \omega)=\widetilde{Z}_{k}\left\{\frac{\Gamma\left(1+\frac{\xi}{2}\right)}{\Gamma\left(\frac{1+\xi}{2}\right) \sqrt{\pi}}\left[\frac{1 / \tau^{1+\xi}}{\left(\widetilde{x}^{2}+(1 / \tau)^{2}\right)^{1+\xi / 2}}\right]\right\}
$$

The normalization constant in Eqn. (11) was derived assuming that $\xi>-1$; otherwise, the integral fails to converge. This makes sense from a physical point of view by looking at the branch cut structure of Eqn. (1): when $\xi \in$ $\mathbb{R} / \mathbb{Z}$, the branch cut consists of a line of complex numbers $z \in(-\infty, 0)$ for $\xi>-1$ and $z \in(-\infty, 0]$ for $\xi<-1$. In the case of the latter, the branch cut includes the point of the Green's function singularity, resulting in a finite quasiparticle lifetime at the Fermi surface and a failure of the quasiparticle paradigm. More drastically, the regime of $\xi<-1$ results in an unphysical (i.e., negative) spectral weight, telling us that the proposal Eqn. (11) is incorrect and no Landau-like quasiparticle can survive for power-law scaling $\xi<-1$. This should be apparent from the form of (3), where the regime of $\xi<-1$ transforms the Fermi surface into a Luttinger surface (i.e., a surface of zeroes of the Green's function as opposed to the Green's function's inverse [52]).

For the case of $\xi>-1$, the spectral density Eqn. (11) displays a sharp peak not unlike what is seen in a traditional Landau-Fermi liquid. The corresponding effective lifetime $\widetilde{\tau}$ can be read off as the width of the Lorentzian:

$$
\widetilde{\tau}=\tau \sqrt{\pi} \frac{\Gamma\left(1+\frac{\xi}{2}\right)}{\Gamma\left(\frac{1+\xi}{2}\right)}
$$




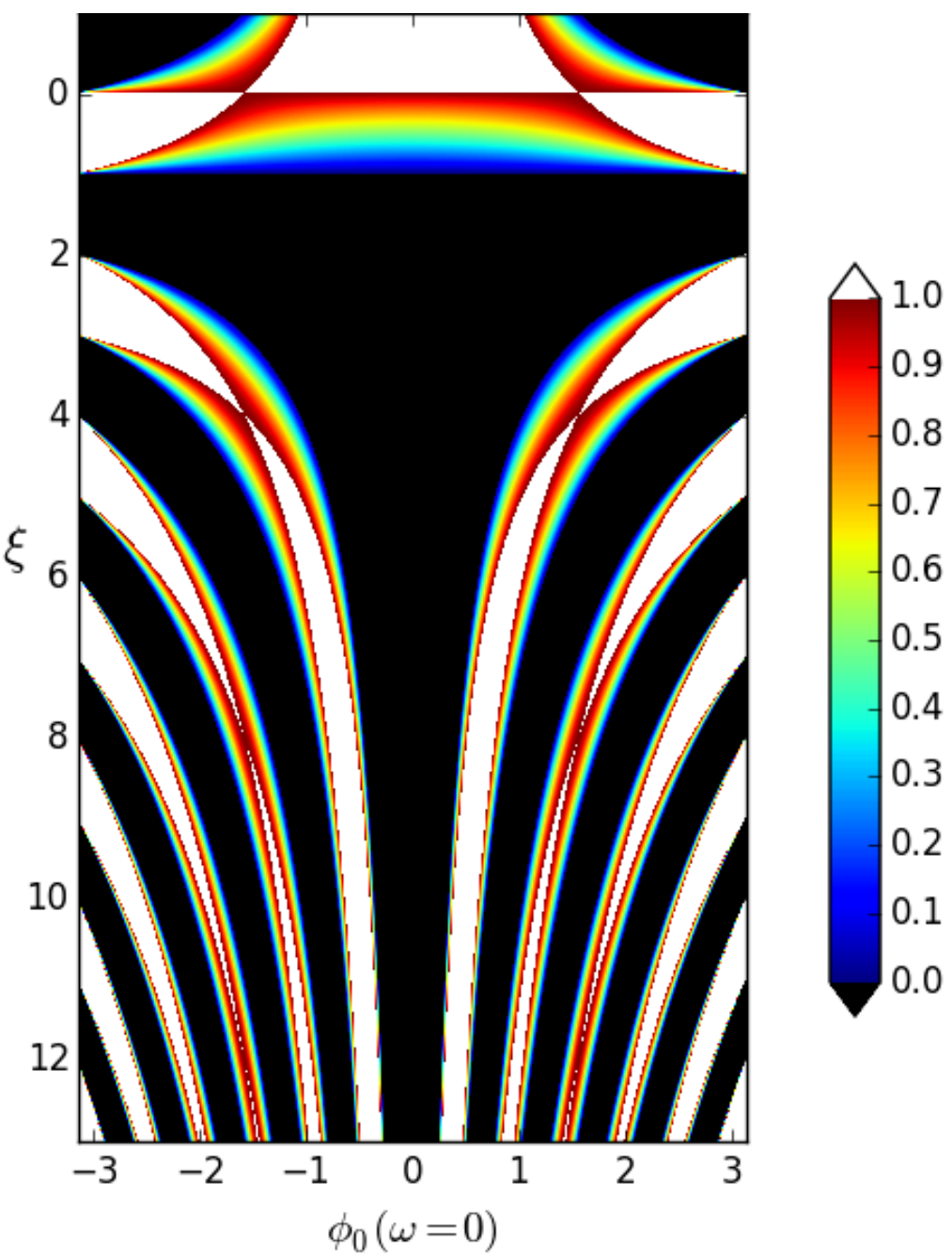

Fig. 1 A density profile of $\widetilde{Z}_{k} / Z_{k}^{\xi+1}$ plotted on a graph of the phase of the $\xi=0$ retarded Green's function $\phi_{0}(\omega=0)$ vs. the power $\xi$ of the power-law Green's function. The colour spectrum represents the regime where $0<\widetilde{Z}_{k} / Z_{k}^{\xi+1} \leq 1$, illustrating where Landau-Fermi liquid theory will unanimously survive in the presence of non-trivial powers $\xi$. The white regions denote where $\widetilde{Z}_{k} / Z_{k}^{\xi+1}>1$, leading us to conclude that only weak Landau-Fermi liquids (i.e., $Z_{k}<<1$ ) can survive in this region. The black regions correspond to $\widetilde{Z}_{k} / Z_{k}^{\xi+1}<$ 0 , and hence the complete breakdown of Landau-Fermi liquid theory. Note that a LandauFermi liquid-like quasiparticle weight is most stable for weak coupling $-1<\xi<1$, while a stable Fermi liquid for $\xi>1$ is only possible for certain non-trivial values of the phase $\phi_{0}(\omega=0)$. 
The above illustrates that the quasiparticle lifetime will remain finite as long as the power-law spectral function is of the form Eqn. (11). Note that $\xi>0$ will result in an apparent increase in the quasiparticle lifetime, while $-1<\xi<0$ results in a reduction.

To gain more insight into the stability or instability of Landau-like excitations in power-law Green's functions, the ratio of the effective weights $\widetilde{Z}_{k} / Z_{k}^{\xi+1}$ is plotted in Fig. 1 as a density plot of $\phi_{0}(\omega=0)$ vs. $\xi$. The conventional result $\phi_{0}(\omega=0)=0$ is shown to exhibit a stable Fermi liquid phase for all $0<\xi<1$, although the quasiparticle weight becomes weaker as $\xi$ approaches unity. For $-1<\xi<0$, the value $\widetilde{Z}_{k} / Z_{k}^{\xi+1}$ is larger than one, implying a nonFermi liquid like phase depending on the initial value of $Z_{k}$. The Fermi liquid ansatz for $\phi_{0}(\omega=0)=0$ is shown to break down for all $\xi>1$ and $\xi<-1$, as the effective quasiparticle weight becomes negative. Interestingly, if we allow the value of the $\xi=0$ retarded Green's function phase to shift from zero to some finite number, we may recover small pockets of Green's functions with powers $\xi>1$ where the effective quasiparticle weight remains non-zero and smaller than one. Physically, this means that Fermi liquid theory is possible for large non-trivial powers of the propagator, provided the imaginary part of the self-energy goes to zero at the same rate as the real part in the vicinity of the Fermi surface. From previous work by the author [42, this implies that the imaginary part of the self energy itself goes as a power law $\Im \Sigma(\mathbf{k}, \omega) \sim \omega^{\alpha}$ with $\alpha<1$. Such self energy behavior has been noted in the pseudogap $\mathrm{Bi}_{2} \mathrm{Sr}_{2} \mathrm{CaCu}_{2} \mathrm{O}_{8+\delta}$, with NMR measurements in the pseudogap phase of the related cuprate $\mathrm{Bi}_{2} \mathrm{Sr}_{2-x} \mathrm{La}_{x} \mathrm{CuO}_{6+\delta}$ suggesting a Fermi liquid-like ground state 36, 53,54. Experimentally, a Landau-Fermi liquid like ground state has also been noted in the pseudogap phase of $\mathrm{HgBa}_{2} \mathrm{CuO}_{4+\delta}$ 55,56,57 and the highfield limit of underdoped $\mathrm{YBa}_{2} \mathrm{Cu}_{3} \mathrm{O}_{6.5}$ [35] and $\mathrm{YBa}_{2} \mathrm{Cu}_{4} \mathrm{O}_{8}$ [58. The calculation of this section therefore provides evidence that, for pseudogap phases with specific values of $\phi_{0}(\omega=0)$, a Landau-Fermi liquid ground state remains stable.

The above discussion leads us to conclude that a stable Landau-Fermi liquid remains possible as long as $\xi>-1$; i.e., the branch-cut singularity resulting from the power-law nature of the propagator does not include the pole of the propagator itself. This statement shouldn't be that surprising; as stated before, branch-cut singularities have been shown to be present in stable Fermi liquids due to the particle-hole continuum [49]. A similar phenomenon is seen in lattices of Sachdev-Ye-Kitaev dots, where the propagator's branch cut singularity can be ignored in the low-temperature Fermi liquid phase [15]. Only when the branch cut engulfs the pole at $G\left(\mathbf{k}_{F}, \omega=0\right)^{-1}=0$ is the Landau-Fermi liquid theory no longer possible, as then the quasiparticle picture breaks down entirely. What is surprising is that, by "tuning" the phase of the retarded Green's function at $\xi=0$, we have the possibility of a stable Fermi liquid for arbitrary values of $\xi$. As we will show in the next section, this results in non-trivial behavior of the fermionic degrees of freedom away from the Fermi surface at non-integer powers. 
III. The status of Luttinger's theorem in power-law liquids.- In its original form, Luttinger's theorem states that the volume of the Fermi surface remains an invariant constant in the presence of interactions $59,60,61$. Originally developed perturbatively for a conventional Fermi liquid, Luttinger's theorem has since been shown to be a robust feature of gapless fermionic systems, haven been shown to be valid for a 1D Tomonaga-Luttinger liquid 62, 63, the Kondo lattice 64, and certain Mott insulators 42. In the context of powerlaw liquids as considered in this work, the status of Luttinger's theorem has been a subject of much debate, with its fragility for powers $0<\xi<1$ already being noted 26 .

In a nutshell, Luttinger's theorem depends solely upon the two following sum rules $65,41,26,42$ :

$$
\begin{gathered}
\frac{i}{2 \pi} \int \frac{d^{d} \mathbf{k}}{(2 \pi)^{d}} \oint_{\mathcal{C}} d \omega \frac{\partial}{\partial \omega} \log (G(\mathbf{k}, \omega))=\frac{N}{2 V} \\
-i \int \frac{d^{d} \mathbf{k}}{(2 \pi)^{d}} \oint_{\mathcal{C}} \frac{d \omega}{2 \pi}\left\{G(\mathbf{k}, \omega) \frac{\partial}{\partial \omega} \Sigma(\mathbf{k}, \omega)\right\}=0
\end{gathered}
$$

Eqn. (13b requires the existence of a well-defined Luttinger-Ward functional, which is equivalent to $\Im \Sigma(\mathbf{k}, \omega) \sim \omega^{\alpha}$, with $\alpha>0$ 66. 42. In the case of strong self energy correlation, we can identify $\alpha \approx \xi+1[26$, leading to $\xi=-1$ as the lower limit for a $\Phi$-derivable $60,67,68$, strongly correlated power-law liquid. Nevertheless, as long as we assume weak self energy correlation, this limit can be relaxed.

Eqn. 13a can be recast in terms of the phase of the retarded Green's function, leading to the constraint that

$$
\frac{N}{2 V}=-\frac{1}{\pi} \int \frac{d^{d} \mathbf{k}}{(2 \pi)^{d}}\{\phi(0)-\phi(-\infty)\}
$$

Hence, for Luttinger's theorem to be obeyed in a generic fermionic system, we must enforce that $\tan [(\phi(0))]=\tan [(\phi(-\infty))]=0$.

The phase $\phi_{\xi}(\omega)$ for non-zero $\xi$ can be found by first writing down the imaginary and real parts of the power-law Green's function, in a similar manner as we did in Eqns. (4) and (6):

$$
\begin{gathered}
\Im G(k, \omega) \sim \Im \Sigma \frac{\cos \left[\xi \phi_{0}(\omega)\right]-\cot \left[\phi_{0}(\omega)\right] \sin \left[\xi \phi_{0}(\omega)\right]}{\left((x-\Re \Sigma(\mathbf{k}, \omega))^{2}+\Im \Sigma(\mathbf{k}, \omega)^{2}\right)^{\xi / 2}} \\
\Re G_{R}(\mathbf{k}, \omega) \sim(x-\Re \Sigma(\mathbf{k}, \omega)) \frac{\cos \left[\xi \phi_{0}(\omega)\right]-\tan \left[\phi_{0}(\omega)\right] \sin \left[\xi \phi_{0}(\omega)\right]}{\left((x-\Re \Sigma(\mathbf{k}, \omega))^{2}+\Im \Sigma(\mathbf{k}, \omega)^{2}\right)^{\xi / 2}}
\end{gathered}
$$

Combining these two results yields the equation connecting the phase of the power-law Green's function with that of the $\xi=0$ propagator: 


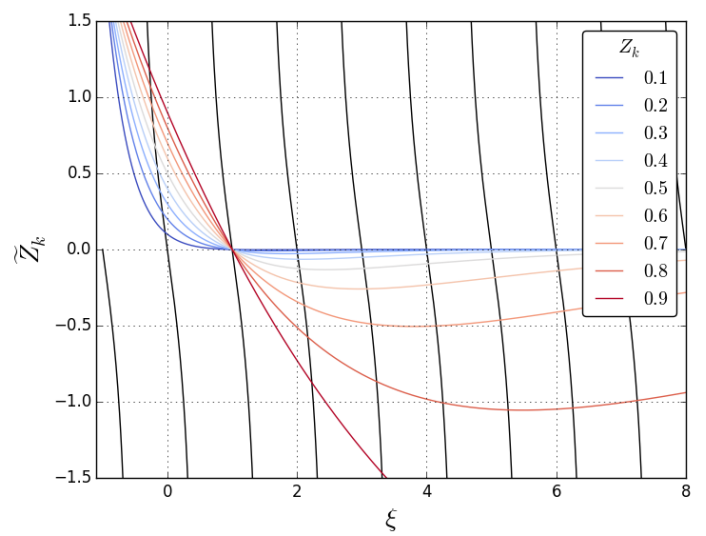

(a)

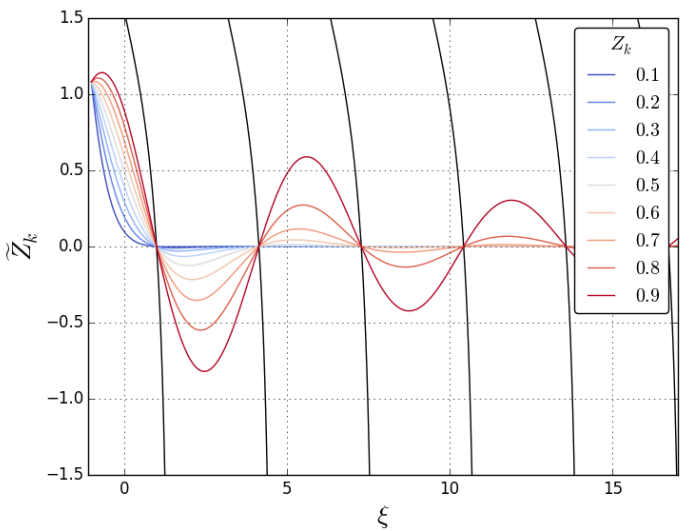

(b)

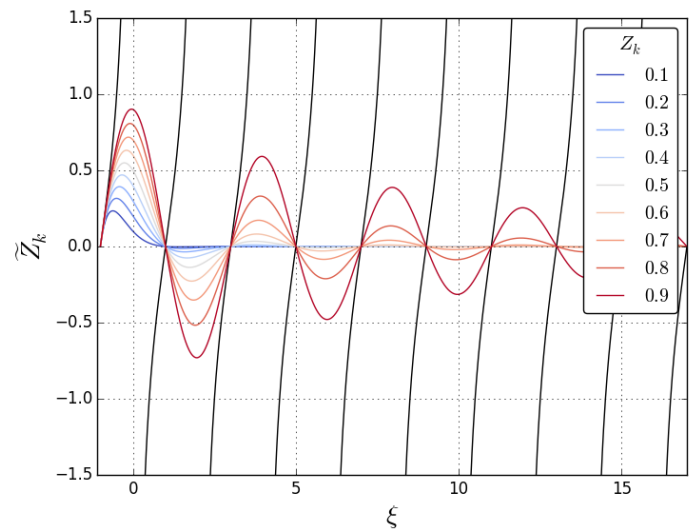

(c)

Fig. 2 Values of the effective quasiparticle weight $\widetilde{Z}_{k}$ vs. $\xi$ (colour) plotted alongside the respective functions given in Eqn. 18 whose zeroes suggest the applicability of Luttinger's theorem (black). In Fig. $2 \mathrm{a}$ the value of the phase is taken as in a conventional LandauFermi liquid. For $\xi>1$ and $\xi \approx-1$, either $\widetilde{Z}_{k}>1$ or $\widetilde{Z}_{k}<0$, and Fermi liquid theory no longer applies. In Fig. $2 \mathrm{~b}$ however, we take $\phi_{0}(0)$ to be some arbitrary value, leading to a weak (but finite) effective quasiparticle weight for larger values of $\xi$. Here I take the phase to be some arbitrary constant; to ensure agreement with the condition Eqn. (17), only phases which yield zeros of $\tan \left[\xi \phi_{0}(\omega)\right]-\tan \left[\phi_{0}(\omega)\right]$ at integer values of $\xi$ lead to the validity of Eqn. 13a. In Fig. 2c we see similar behavior as in Fig. 2a although this result is highly improbable as it violates causality. In all three cases, zeros of the functions defined in Eqn. (18) occur when $\widetilde{Z}_{k}=0$, leading us to conclude that Luttinger's theorem and Landau-Fermi liquid theory are always incompatible for power-law fluids. 


$$
\tan \left[\phi_{\xi}(\omega)\right]=\frac{\tan \left[\phi_{0}(\omega)\right] \cos \left[\xi \phi_{0}(\omega)\right]-\sin \left[\xi \phi_{0}(\omega)\right]}{\cos \left[\xi \phi_{0}(\omega)\right]-\tan \left[\phi_{0}(\omega)\right] \sin \left[\xi \phi_{0}(\omega)\right]}
$$

The condition that $\phi_{0}(-\infty)=\pi$ is a direct consequence of causality, and hence a universal constraint on any fermionic system [69,42. In the context of powerlaw Green's functions, we simply replace the condition of $-\infty$ with some UV cutoff $-\Delta$ which we assume to be reasonably large such that $\phi_{0}(-\Delta)=\pi$ to reasonable precision. Therefore, we can see that

$$
\tan \left[\phi_{\xi}(-\Delta)\right]=-\tan [\xi \pi]
$$

This is guaranteed to equal zero for integer values of the power $\xi$, which agrees with the result for $\xi=0$ when we take $\Delta \rightarrow \infty$.

For the zero-frequency case, the validity of Luttinger's theorem depends upon the limiting value of $\tan \left[\phi_{0}(0)\right]$, as outlined below:

$$
\tan \left[\phi_{0}(0)\right]= \begin{cases}0, & -\tan [n \pi \xi]=0, \quad \xi \neq 1 \\ \text { const, } & \tan \left[\xi \phi_{0}(0)\right]=\tan \left[\phi_{0}(0)\right], \quad \xi \neq 1+\frac{n}{m+1 / 2} \\ \infty, & -\cot [\pi(m+1 / 2) \xi]=0, \quad \xi \neq 1\end{cases}
$$

where $m, n \in \mathbb{Z}$. The first limit in the above is the common one encountered when $\xi=0$, and corresponds to an imaginary part of the self energy which goes to zero faster than the real part; i.e., $\Im \Sigma(\mathbf{k}, \omega) \sim \omega^{\alpha}, \alpha>1$ [42]. The second term corresponds to the real and imaginary portions of the self energy going to zero at the same rate, i.e. $\Im \Sigma(\mathbf{k}, \omega) \sim \omega^{\alpha}, 0<\alpha<1$, while the final limit corresponds to the imaginary portion either going to a constant or going to zero slower than the real portion. As the final of these conditions violates causality, the first two limits are of greatest physical concern. The case when $\xi=1$ is special in that $\tan \left[\phi_{0}(0)\right]$ can be any arbitrary value for Luttinger's theorem to remain valid.

As the effective quasiparticle weight $\widetilde{Z}_{k}$ is also dependent on the phase $\phi_{0}(\omega=0)$, we can check the compatibility of the above relations with the existence of a well-defined Landau quasiparticle. In Fig. 2, I plot $\widetilde{Z}_{k}$ alongside the underlying conditions for Luttinger's Theorem given in Eqn. 18 vs. $\xi$. Note that, if we take values of $\phi_{0}(0)$ for the case of $\left|\tan \left[\phi_{0}(0)\right]\right| \in \mathbb{R}_{>0}$ such that $\tan \left[\xi \phi_{0}(0)\right]-\tan \left[\phi_{0}(0)\right]=0$ only for $\xi \in \mathbb{Z}$, then we can see that such a limit is compatible with the condition $\tan \left[\phi_{\xi}(-\Delta)\right]=0$; i.e., by Eqn. (17), Luttinger's theorem is only valid at integer values of $\xi$. This agrees with the work of Limtragool et. al. [26], where Luttinger's theorem is strongly violated for $-1<\xi<1, \xi \neq 0$. For the case $\xi=1$, Luttinger's theorem is always applicable, irrespective of the behavior of the self energy, although the effective quasiparticle weight will always be zero.

It is important to note that, for all cases examined in the above excluding $\tan \left[\phi_{0}(0)\right]=0$ with $\xi=0$, Luttinger's theorem is incompatible with a finitevalued effective quasiparticle weight. Although this result is surprising, it is not 
unheard of; the sharp peaks in underdoped samples of $\mathrm{Bi}_{2} \mathrm{Sr}_{2-x} \mathrm{La}_{x} \mathrm{CuO}_{6+\delta}[70$, 71] and the small Fermi surfaces in the underdoped phases of $\mathrm{HgBa}_{2} \mathrm{CuO}_{4+\delta}$ [72], $\mathrm{YBa}_{2} \mathrm{Cu}_{4} \mathrm{O}_{8}\left[58\right.$, and $\mathrm{YBa}_{2} \mathrm{Cu}_{3} \mathrm{O}_{6.5}[35$, have led to the postulate that the non-superconducting pseudogap phase of the cuprate superconductors (in the absence of Fermi arcs) is an exotic variant of the traditional Fermi liquid where fermionic degrees of freedom deep in the Fermi surface are lost in the presence of strong interactions $[36,37,38,39,40$. As stated previously, the Fermi liquid phase is stable for the pseudogap phase characterized by $\Im \Sigma(\mathbf{k}, \omega) \sim \omega^{\alpha}$ with $\alpha<1$, but only if the phase of the retarded Green's function at $\xi=0$ is non-trivial. Hence, if such an exotic metallic state in the pseudogap is truly described by power-law Green's functions, the work presented above shows that the incompatibility of Luttinger's theorem and Fermi liquid theory is not a coincidence, but is instead a deep physical law that must be obeyed for any scale-invariant electronic propagator.

IV. Discussion.- I have shown that Landau-Fermi liquid theory is possible in the presence of power-law scaling of the retarded Green's function as long as said scale invariance does not change the underlying topology of the Fermi surface. The power-law scaling can be recast in the form of a rescaled quasiparticle weight, which becomes zero for powers $\xi>1$ unless the phase of the retarded Green's function at unity power takes on non-trivial values. It is then shown that Luttinger's theorem breaks down for all cases of a stable Fermi liquid solution, excluding the conventional $\xi=0$ solution. This agrees with recent experiments on the pseudogap phase of cuprate superconductors, where a Fermi liquid-like phase is seen in the presence of possible power-law scaling.

It is interesting to compare this result with the contradictory prediction that scale-invariance always leads to the breakdown of particle-like excitations in the IR [29,30]. Such an "unparticle" regime is usually characterized by the unparticle dimension $d_{U}$, which can be related to our coefficient $\xi$ via the relation $\xi=\frac{d-1}{2}-d_{U}$, where $d$ is the physical dimension. The conditions of unitarity constrain $d_{U}>\frac{d-1}{2}$ [73,74, leading to a power-law scaling of $-1<$ $\xi<0$ being compatible with both the predictions of unparticle physics and a well-defined Landau quasiparticle in $d \geq 2$. The existence of such a discrepancy can be justified in the context of quantum critical phenomena by recalling the effects of particle-hole asymmetry on the two-point time-dependent correlation function of the order parameter [75]. If quantum critical scaling induces a scaleinvariant form of the Green's function as given in Eqn. (1), then the system will experience severe particle-hole asymmetry either below or above the Fermi surface, and hence the emergent bosonic order parameter from the fermionic degrees of freedom (from Cooper pairing or otherwise) will be particle-hole asymmetric as well. Because the order parameter field for a system defined by Eqn. (1) is particle-hole asymmetric, the spatial correlation length will be finite, resulting in a finite scattering cross section and the possibility for dominant Fermi-liquid-like behavior.

Finally, the above might have important implications in the context of interacting, itinerant Majorana fermions, which have been shown to exhibit a 
finite discontinuity in the momentum distribution function while also displaying a small Fermi surface [76,77]. In the context of a Landau-Majorana liquid 78, the presence of severe particle-hole asymmetry has been shown to lead to robust stability of the Landau quasiparticle state, which agrees with the work presented in this paper. It would be an interesting direction of future research to pursue the similarities between power-law liquids and Landau-Fermi-like Majorana liquids, and explore how such a theory can further elucidate the non-trivial physics of the cuprate pseudogap phase.

Acknowledgements.-The author thanks Kevin Bedell, Krastan Blagoev, Matthew Gochan, and Kridsanaphong Limtragool for useful comments and suggestions.

\section{References}

1. Hertz J A 1976 Phys. Rev. B 14(3) 1165-1184 URL https://link.aps.org/doi/10. 1103/PhysRevB.14.1165

2. Millis A J 1993 Phys. Rev. B 48(10) 7183-7196 URL https://link.aps.org/doi/10. 1103/PhysRevB.48.7183

3. Schofield A J 1999 Contemporary Physics 40 95-115 URL https://www.tandfonline. com/doi/abs/10.1080/001075199181602

4. Sachdev S 2011 Quantum Phase Transitions (Cambridge, CB2 8BS, United Kingdom: Cambridge University Press) ISBN 978-0-521-51468-2

5. Su Y H and Lu H T 2017 Frontiers of Physics 13137103 URL https://doi.org/10. 1007/s11467-017-0734-2

6. Ando Y, Komiya S, Segawa K, Ono S and Kurita Y 2004 Phys. Rev. Lett. 93(26) 267001 URL https://link.aps.org/doi/10.1103/PhysRevLett.93.267001

7. Hussey N E 2008 Journal of Physics: Condensed Matter 20123201 URL https:// iopscience.iop.org/article/10.1088/0953-8984/20/12/123201

8. Cooper R A, Wang Y, Vignolle B, Lipscombe O J, Hayden S M, Tanabe Y, Adachi T, Koike Y, Nohara M, Takagi H, Proust C and Hussey N E 2009 Science $323603-607$ ISSN 0036-8075 URL https://science.sciencemag.org/content/323/5914/603

9. Chien T R, Wang Z Z and Ong N P 1991 Phys. Rev. Lett. 67(15) 2088-2091 URL https://link.aps.org/doi/10.1103/PhysRevLett.67.2088

10. Tyler A and Mackenzie A 1997 Physica C: Superconductivity 282-287 1185 - 1186 ISSN 0921-4534 proceedings of the International Conference on Materials and Mechanisms of Superconductivity High Temperature Superconductors V URL http://www . sciencedirect.com/science/article/pii/S092145349700751X

11. Zhang Y, Ong N P, Xu Z A, Krishana K, Gagnon R and Taillefer L 2000 Phys. Rev. Lett. 84(10) 2219-2222 URL https://link.aps.org/doi/10.1103/PhysRevLett.84.2219

12. Hartnoll S A and Karch A 2015 Phys. Rev. B 91(15) 155126 URL https://link.aps. org/doi/10.1103/PhysRevB.91.155126

13. Karch A 2014 Journal of High Energy Physics 2014 URL https://link.springer. com/article/10.1007/JHEP06 (2014) 140

14. Patel A A, McGreevy J, Arovas D P and Sachdev S 2018 Phys. Rev. X 8(2) 021049 URL https://link.aps.org/doi/10.1103/PhysRevX.8.021049

15. Chowdhury D, Werman Y, Berg E and Senthil T 2018 Phys. Rev. X 8(3) 031024 URL https://link.aps.org/doi/10.1103/PhysRevX.8.031024

16. Patel A A and Sachdev S 2019 Phys. Rev. Lett. 123(6) 066601 URL https://link. aps.org/doi/10.1103/PhysRevLett.123.066601

17. La Nave G, Limtragool K and Phillips P W 2019 Rev. Mod. Phys. 91(2) 021003 URL https://link.aps.org/doi/10.1103/RevModPhys.91.021003

18. Phillips P W and La Nave G Springer Proceedings in Mathematics and Statistics, 2020 (Preprint arXiv:1911.05750) URL https://arxiv.org/abs/1911.05750 
19. Landau L 1956 JETP 3(6) 1058 URL http://www.jetp.ac.ru/cgi-bin/e/index/e/3/ $6 / \mathrm{p} 920 ? \mathrm{a}=1$ ist

20. Migdal A 1957 JETP 5(2) 399 URL http://www.jetp.ac.ru/cgi-bin/e/index/e/5/ $2 / \mathrm{p} 333$ ?a=list

21. Galitskii V and Migdal A 1958 JETP 7(1) 139 URL http://www.jetp.ac.ru/cgi-bin/ e/index/e/7/1/p96?a=list

22. Abrikosov A A, Dzyaloshinski I E and Gor'kov L 1975 Methods of Quantum Field Theory in Statistical Physics (Mineola, New York: Dover Publications) ISBN 978-0486-63228-5

23. Anderson P W 2008 Phys. Rev. B 78(17) 174505 URL https://link.aps.org/doi/10. 1103/PhysRevB.78.174505

24. Anderson P W and Casey P A 2009 Phys. Rev. B 80(9) 094508 URL https://link. aps.org/doi/10.1103/PhysRevB.80.094508

25. Reber T et al. 2015 (Preprint arXiv:1509.01611v1) URL https://arxiv.org/abs/ 1509.01611

26. Limtragool K, Leong Z and Phillips P W 2018 SciPost Phys. 5(5) 49 URL https: //scipost.org/10.21468/SciPostPhys.5.5.049

27. Phillips P W, Langley B W and Hutasoit J A 2013 Phys. Rev. B 88(11) 115129 URL https://link.aps.org/doi/10.1103/PhysRevB.88.115129

28. Leong Z, Setty C, Limtragool K and Phillips P W 2017 Phys. Rev. B 96(20) 205101 URL https://link.aps.org/doi/10.1103/PhysRevB.96.205101

29. Georgi H 2007 Phys. Rev. Lett. 98(22) 221601 URL https://link.aps.org/doi/10. 1103/PhysRevLett.98.221601

30. Georgi H 2007 Physics Letters B 650275 - 278 ISSN 0370-2693 URL http://www. sciencedirect.com/science/article/pii/S0370269307006296

31. Gochan M P, Li H and Bedell K S 2019 Journal of Physics Communications 3065008 URL https : //iopscience.iop.org/article/10.1088/2399-6528/ab292b/meta

32. Wölfle P and Rosch A 2007 Journal of Low Temperature Physics $147165-177$ URL https://link.springer.com/article/10.1007/s10909-007-9308-y

33. Wölfle P and Abrahams E 2011 Phys. Rev. B 84(4) 041101 URL https://link.aps. org/doi/10.1103/PhysRevB.84.041101

34. Abrahams E and Wölfle P 2012 Proceedings of the National Academy of Sciences 109 3238-3242 ISSN 0027-8424 URL https://www.pnas.org/content/109/9/3238

35. Doiron-Leyraud N et al. 2007 Nature 447 565?568 URL https://www.nature.com/ articles/nature05872

36. Mei J W, Kawasaki S, Zheng G Q, Weng Z Y and Wen X G 2012 Phys. Rev. B 85(13) 134519 URL https://link .aps.org/doi/10.1103/PhysRevB.85.134519

37. Senthil T, Sachdev S and Vojta M 2003 Phys. Rev. Lett. 90(21) 216403 URL https: //link.aps.org/doi/10.1103/PhysRevLett.90.216403

38. Punk M, Allais A and Sachdev S 2015 Proceedings of the National Academy of Sciences 112 9552-9557 URL https://www.pnas.org/content/112/31/9552

39. Sachdev S 2018 Reports on Progress in Physics 82014001 URL https://iopscience. iop.org/article/10.1088/1361-6633/aae110

40. Moon E G and Sachdev S 2011 Phys. Rev. B 83(22) 224508 URL https://link.aps. org/doi/10.1103/PhysRevB.83.224508

41. Blagoev K B and Bedell K S 1997 Phys. Rev. Lett. 79(6) 1106-1109 URL https: //link.aps.org/doi/10.1103/PhysRevLett.79.1106

42. Heath J T and Bedell K S 2020 New J. Phys. 22 063011 ISSN 1367-2630 URL https: //iopscience.iop.org/article/10.1088/1367-2630/ab890e

43. Haldane F D M 1981 Journal of Physics C: Solid State Physics 14 2585-2609 URL https://iopscience.iop.org/article/10.1088/0022-3719/14/19/010/meta

44. Wen X G 1990 Phys. Rev. B 42(10) 6623-6630 URL https://link.aps.org/doi/10. 1103/PhysRevB.42.6623

45. Voit J 1995 Reports on Progress in Physics 58 977-1116 URL https://iopscience. iop.org/article/10.1088/0034-4885/58/9/002

46. Gonzálex J, Martín-Delgado M A, Sierra G and Vozmediano A H 1995 Quantum Electron Liquids and high-T $T_{c}$ Superconductivity (Heidelberger Pl. 3, 14197 Berlin, Germany: Springer-Verlag) ISBN 3-540-60503-7 
47. Sólyom J 1979 Adv. Phys. 28 201-303 ISSN 0001-8732 URL https://www.tandfonline. com/doi/abs/10.1080/00018737900101375

48. Ruckenstein A and Varma C 1991 Physica C: Superconductivity 185-189 134 - 140 ISSN 0921-4534 URL http://www.sciencedirect.com/science/article/pii/ 0921453491919624

49. Shekhter A and Finkel'stein A M 2006 Proc. Natl. Acad. Sci. U.S.A. $10315765-15769$ ISSN 0027-8424 URL https://www.pnas.org/content/103/43/15765

50. Dzyaloshinskii I E and Larkin A I 1974 JETP Letters 38(1) 201 URL http://www. jetp.ac.ru/cgi-bin/e/index/e/38/1/p202?a=list

51. Bares P A and Wen X G 1993 Phys. Rev. B 48(12) 8636-8650 URL https://link. aps.org/doi/10.1103/PhysRevB.48.8636

52. Dzyaloshinskii I 2003 Phys. Rev. B 68(8) 085113 URL https://link.aps.org/doi/10. 1103/PhysRevB.68.085113

53. Zheng G q, Kuhns P L, Reyes A P, Liang B and Lin C T 2005 Phys. Rev. Lett. 94047006 ISSN 1079-7114 URL https://link.aps.org/doi/10.1103/PhysRevLett.94.047006

54. Kawasaki S, Lin C, Kuhns P L, Reyes A P and Zheng G q 2010 Phys. Rev. Lett. 105(13) 137002 URL https://link.aps.org/doi/10.1103/PhysRevLett.105.137002

55. Mirzaei S I, Stricker D, Hancock J N, Berthod C, Georges A, van Heumen E, Chan M K, Zhao X, Li Y, Greven M, Barišić N and van der Marel D 2013 Proc. Natl. Acad. Sci. U.S.A. 110 5774-5778 ISSN 0027-8424 URL http://www.pnas.org/content/110/ 15/5774. abstract

56. Barišić N, Chan M K, Li Y, Yu G, Zhao X, Dressel M, Smontara A and Greven M 2013 Proc. Natl. Acad. Sci. U.S.A. 110 12235-12240 ISSN 0027-8424 URL http:// ww. pnas.org/content/110/30/12235.abstract

57. Chan M K, Veit M J, Dorow C J, Ge Y, Li Y, Tabis W, Tang Y, Zhao X, Barišić N and Greven M 2014 Phys. Rev. Lett. 113(17) 177005 URL https://link.aps.org/doi/10. 1103/PhysRevLett.113.177005

58. Proust C, Vignolle B, Levallois J, Adachi S and Hussey N E 2016 Proc. Natl. Acad. Sci. U.S.A. 113 13654-13659 ISSN 0027-8424 URL http://www.pnas.org/content/113/48/ 13654.abstract

59. Kohn W and Luttinger J M 1960 Phys. Rev. 118(1) 41-45 URL https://link.aps. org/doi/10.1103/PhysRev.118.41

60. Luttinger J M and Ward J C 1960 Phys. Rev. 118(5) 1417-1427 URL https://link. aps.org/doi/10.1103/PhysRev.118.1417

61. Luttinger J M 1960 Phys. Rev. 119(4) 1153-1163 URL https://link.aps.org/doi/ 10.1103/PhysRev.119.1153

62. Blagoev K B and Bedell K S 1997 Phys. Rev. Lett. 79(6) 1106-1109 URL https: //link.aps.org/doi/10.1103/PhysRevLett.79.1106

63. Yamanaka M, Oshikawa M and Affleck I 1997 Phys. Rev. Lett. 79(6) 1110-1113 URL https://link.aps.org/doi/10.1103/PhysRevLett.79.1110

64. Oshikawa M 2000 Phys. Rev. Lett. 84(15) 3370-3373 URL https://link.aps.org/doi/ 10.1103/PhysRevLett.84.3370

65. Giuliani G F and Vignale G 2005 Quantum Theory of the Electron Liquid (The Edinburgh Building, Cambridge CB2 2RU, UK: Cambridge University Press) ISBN 978-0$521-82112-6$

66. Stanescu T D, Phillips P and Choy T P 2007 Phys. Rev. B 75(10) 104503 URL https: //link.aps.org/doi/10.1103/PhysRevB.75.104503

67. Baym G 1962 Phys. Rev. 127(4) 1391-1401 URL https://link.aps.org/doi/10.1103/ PhysRev.127.1391

68. Cornwall J M, Jackiw R and Tomboulis E 1974 Phys. Rev. D 10(8) 2428-2445 URL https://link.aps.org/doi/10.1103/PhysRevD.10.2428

69. Tremblay A M T 2008 Lecture notes for Cifar-PiTP International Summer School on Numerical Methods for Correlated Systems in Condensed Matter URL https://pitp. phas.ubc.ca/confs/sherbrooke/archives.html

70. Meng J et al. 2009 Phys. Rev. B 79(2) 024514 URL https://link.aps.org/doi/10. 1103/PhysRevB.79.024514

71. Meng J et al. 2009 Nature 462 335-338 URL https://www.nature.com/articles/ nature08521 
72. Chan M K, McDonald R D, Ramshaw B J, Betts J B, Shekhter A, Bauer E D and Harrison N 2020 Proc. Natl. Acad. Sci. U.S.A. 117 9782-9786 ISSN 0027-8424 URL http://www .pnas.org/content/117/18/9782.abstract

73. Minwalla S 1998 Advances in Theoretical and Mathematical Physics 2(4) 783-851 URL https://www.intlpress.com/site/pub/pages/journals/items/atmp/ content/vols/0002/0004/a004/

74. Limtragool K, Setty C, Leong Z and Phillips P W 2016 Phys. Rev. B 94(23) 235121 URL https://link.aps.org/doi/10.1103/PhysRevB.94.235121

75. Cha M C and Ortiz G 2008 Phys. Rev. B 77(16) 165124 URL https://link.aps.org/ doi/10.1103/PhysRevB.77.165124

76. Baskaran G Majorana fermi sea in insulating smb6: A proposal and a theory of quantum oscillations in kondo insulators Invited Talk at the Workshop 'Concepts and Discovery in Quantum Matter', 12-15th July 2015, Cavendish Laboratory, Cambridge University, UK URL https://arxiv.org/abs/1507.03477

77. Heath J T and Bedell K S 2019 Journal of Physics A: Mathematical and Theoretical 52315001 URL https://iopscience.iop.org/article/10.1088/1751-8121/ab2a86

78. Heath J and Bedell K 2019 Under review (Preprint arXiv:1903.00619) URL https: //arxiv.org/abs/1903.00619 